\title{
Efecto de un programa de atención primaria de salud en pacientes mexicanos con enfermedades crónicas no trasmisibles
}

\author{
Arturo Alejandro Domínguez López ${ }^{1}$, Johannes Oudhof van Barneveld ${ }^{2}$, \\ Norma Ivonne González-Arratia López Fuentes ${ }^{3}$, Mirta Margarita Flores Galaz ${ }^{4}$ \\ Universidad Autónoma del Estado de México ${ }^{1,2,3}$, \\ Universidad Autónoma de Yucatán ${ }^{4}$
}

Las Enfermedades Crónicas No Transmisibles (ECNT) son padecimientos de larga duración y su evolución es lenta. Actualmente el número de casos va en ascenso y representan la primera causa de muerte a nivel mundial. Los tratamientos suelen ser complejos y si la población no comienza a hacerse responsable, tomando en cuenta los hábitos saludables, las consecuencias serán mayores para los sistemas de salud. Por ello se elaboró un programa con base en el enfoque de Atención Primaria de Salud, se sometió a análisis por expertos y se estableció una investigación con diseño pretest-postest que se llevó a cabo en los municipios ubicados en el valle de Toluca, en el centro del país con 188 pacientes con ECNT.

Palabras clave: Enfermedades crónicas, Programa de salud, Atención primaria, autocuidado y adherencia terapéutica.

Effect of a primary health care program in Mexican patients with chronic non-communicable diseases

Noncommunicable Chronic Diseases (CNCD) are long-term conditions and their evolution is slow. Currently the number of cases is on the rise and represent the leading cause of death worldwide. The treatments are usually complex and if the population does not begin to take responsibility, taking into account the healthy habits, the consequences will be greater for the health systems. For this reason, a program based on the Primary Health Care approach was prepared, submitted to expert analysis and a pretest-posttest design

1 Doctorante de Ciencias de la Salud por la Universidad Autónoma del Estado de México. Dirección postal: 3 a Cerrada de Lomas Estrella 102-A. C.P. 51355. Zinacantepec, Estado de México, México. Contacto: dandrosh@hotmail.com https://orcid.org/0000-0003-1435-8559

2 Integrante del Sistema Nacional de Investigadores en México. Universidad Autónoma del Estado de México, México. Dirección postal: Filiberto Gómez s/n, Barrio de Tlacopa, C.P. 50010. Toluca de Lerdo, Estado de México, México. Contacto: hansovb@hotmail.com https://orcid.org/0000-0003-1265-3052

3 Integrante del Sistema Nacional de Investigadores en México. Universidad Autónoma del Estado de México, México. Dirección postal: Filiberto Gómez s/n, Barrio de Tlacopa, C.P. 50010. Toluca de Lerdo, Estado de México, México. Contacto: nigalf@yahoo.com.mx https://orcid.org/0000-0003-0497-119X

4 Integrante del Sistema Nacional de Investigadores en México. Universidad Autónoma de Yucatán. Dirección postal: Km 1 Carretera Mérida, Tizimin, Cholul C.P. 97305. Mérida, Yucatán, México. Contacto: fgalaz@correo.uady.mx https://orcid.org/0000-0002-9971-8404 
research was established in the municipalities located in the Toluca Valley, in the center of the country with 188 patients with CNCD.

Keywords: Chronic diseases, Health Program, Primary care, self-care \& therapeutic adherence.

Efeito de um programa de atençáo primária à saúde em pacientes mexicanos com doenças crônicas não transmissíveis

As Doenças Crônicas Não Transmissíveis (DCNT) são condiçóes de longo prazo e sua evoluçấo é lenta. Atualmente, o número de casos está em ascenso e representa a principal causal de mortes em todo o mundo. Os tratamentos geralmente são complexos e se a população não começa a assumir responsabilidade, tomando em conta os hábitos saudáveis, as consequências serão maiores para os sistemas de saúde. Por isso, foi elaborado um programa baseado na abordagem da Atenção Primária à Saúde, submetido à análise de especialistas e uma pesquisa pré-teste-pós-teste foi estabelecida nos municípios localizados no Vale do Toluca, no centro do país com 188 pacientes com DCNT.

Palavras-chave: Doenças crônicas, programa de saúde, atenção básica, autocuidado e adesão terapêutica.

Effet d'un programme de soins de santé primaires chez les patients mexicains atteints de maladies chroniques non transmissibles

Les maladies chroniques non transmissibles sont des conditions à long terme et leur évolution est lente. Actuellement, le nombre de cas est en augmentation et représente la principale cause de décès dans le monde. Les traitements sont généralement complexes et si la population ne commence pas à assumer ses responsabilités, compte tenu des habitudes saines, les conséquences seront plus lourdes pour les systèmes de santé. Pour cette raison, un programme basé sur l'approche des soins de santé primaires a été préparé, soumis à une analyse par des experts et une étude de conception pré-test / post-test a été établie dans les municipalités situées dans la vallée de Toluca, centre du pays avec 188 patients atteints de CNCD. Mots clés: maladies chroniques, programme de santé, soins primaires, soins personnels et observance thérapeutique. 
Las enfermedades crónicas no trasmisibles (ECNT) son un grupo heterogéneo de enfermedades que tienden a ser de larga duración, resultan de la combinación de factores genéticos, fisiológicos y ambientales (Rivera et al., 2014). Son actualmente de los mayores retos a los que se enfrenta el sistema de salud debido a varios motivos: la creciente cantidad de personas afectadas en México, la contribución a la mortalidad y la necesidad de utilizar tratamientos complejos para la mejora del nivel de salud del sector de la población que vive con padecimientos tales como diabetes mellitus tipo 2, hipertensión arterial, enfermedades del corazón, síndrome metabólico, obesidad, entre otras (Mireles et al., 2017).

Estas enfermedades, especialmente la diabetes mellitus tipo 2, obesidad en cualquiera de sus tipos, hipertensión arterial sistémica, y la enfermedad pulmonar obstructiva crónica (EPOC), no solamente están en aumento, sino que son responsables de un gran número de muertes a nivel mundial y nacional ocasionando alrededor de 36 de los 57 millones de fallecidos por enfermedades cardiovasculares en todo el mundo. Más del $80 \%$ de las muertes por ECNT se producen en países de ingresos medios y bajos, como México (Lizaraso \& Ruiz, 2016). Actualmente el sistema de salud pública se encuentra saturado y no se puede dar solución a la problemática de los pacientes solamente desde el enfoque asistencial. Los decesos a causa de estas son consecuencia de un proceso iniciado décadas antes.

El tratamiento farmacológico y asistencial es necesario para reducir a corto plazo las consecuencias de las ECNT. Sin embargo, esta atención dentro de las instituciones de salud suele ser costosa, tardía y poco satisfactoria en muchos casos (Chauca et al., 2018). Sin embargo, su evolución puede ser modificada mediante acciones que cambien el curso clínico de las condiciones que determinan su incidencia, sobre todo en el caso de la diabetes, obesidad, enfermedad obstructiva 
crónica, tabaquismo, síndrome metabólico y padecimientos cardiovasculares (Jordan et al., 2015).

El manejo de las ECNT tiene su fundamento en distintos principios, como el enfoque de atención primaria de salud, que implica un proceso educativo para que el paciente entienda su padecimiento y establezca cambios significativos focalizados en las conductas saludables, acuda puntualmente a las evaluaciones médicas y utilice correctamente los múltiples fármacos para el control de los síntomas propios de su enfermedad. Todo ello con la participación del equipo de salud, su familia y el entorno donde se desenvuelve (Strong et al., 2015). No obstante, las instituciones de salud pública en México no cuentan con la infraestructura para tal desafío pues se requiere de la participación de diversos profesionales de la salud como psicólogos, nutriólogos, preparadores físicos, enfermeros especializados, entre otros. Y debido a ello la participación del personal de salud se limita al tratamiento de las complicaciones presentes, sin prevenir otros factores de riesgo que conllevan las ECNT, ni se toman en cuenta las expectativas ni el compromiso del paciente para observar su tratamiento, lo que hace que el sistema de salud sea ineficiente y costoso porque el paciente no comprende los objetivos de la terapia (Rivera et al., 2015). A lo anterior se suman factores culturales que limitan la adherencia terapéutica y afectan el bienestar del individuo que vive con una ECNT (Etzwiler, 2017).

Es preciso el desarrollo de programas encaminados a mejorar conductas de autocuidado, de lo contrario la mayor parte del presupuesto se destinará al pago de las complicaciones e incapacidades prematuras, como lo explica última Encuesta Nacional de Salud y Nutrición en México (Romero et al., 2014). Es necesario un nuevo planteamiento en investigaciones que pertenezcan al campo de las ciencias de la salud desde un enfoque de atención primaria que implique la búsqueda del bienestar desde la prevención de las enfermedades, que evite que la población enferme hasta el cambio de perspectiva en las personas que ya están diagnosticadas con alguna ECNT, buscando crear una nueva perspectiva reflexiva por parte del enfermo crónico (Vignolo et al., 2017). Investigaciones de este estilo pueden ser precursoras también 
de programas educativos en donde el estudiante de pregrado y posgrado tenga una actitud crítica ante el sistema de salud actual y el abordaje clínico actual que no dota al paciente de las herramientas necesarias para evitar que la población enferme.

El autocuidado es una variable imprescindible para tomar en cuenta para evitar consecuencias en la salud del paciente con ECNT, pues se ha demostrado que el esfuerzo de los equipos multidisciplinarios que establecen el autocuidado en los pacientes con enfermedades crónico-degenerativas da como resultado el control de parámetros de salud como son la glucosa, presión sanguínea, colesterol, triglicéridos, ácido úrico, entre otros. Esto a su vez se traduce en la disminución de complicaciones en dichas personas (Gallardo \& Monroy, 2016).

Asimismo, se ha explorado, a través de modelos transteóricos, la necesidad que tienen los pacientes que sufren de ECNT de fomentar la adherencia terapéutica que implica cinco etapas: la precontemplación, la contemplación, la preparación, la acción y manutención. Los pacientes que completan todas las etapas tienden a conservar la salud por más tiempo (García \& Lucas, 2013). De la misma manera, se ha hecho necesaria la medición del bienestar en personas con ECNT, pues la terapéutica en general tiene como objetivo devolver la salud a un individuo, misma que implica el bienestar en tres dimensiones: física, psíquica y social (Alpi et al., 2016).

También se ha señalado la necesidad de estudiar el apoyo familiar en los pacientes con enfermedades crónicas, pues la falta de este da como resultado el estrés para el paciente y sus cuidadores además de que la institucionalización es más frecuente cuando se carece de la colaboración de la familia para proporcionar los cuidados más esenciales (Alpi et al., 2016).

Del mismo modo, se ha comprobado que las personas que viven con ECNT y hacen uso de estrategias para afrontar el dolor crónico recuperan más rápidamente su salud física y psicológica. La utilización combinada de estas estrategias, además, motiva al paciente a la rehabilitación permitiéndole recuperar la movilidad y mejorando su calidad de vida (Jiménez et al., 2017). 
Por ello, el objetivo general de este estudio fue medir el efecto de un programa de educación en salud basado en el enfoque de atención primaria de salud, destinado a la mejora su autocuidado, adherencia al tratamiento, apoyo familiar, afrontamiento al dolor crónico y bienestar subjetivo en pacientes del Estado de México, con enfermedades crónicas no trasmisibles. Por todo lo anterior se establece la siguiente hipótesis de investigación: Existen diferencias estadísticamente significativas antes y después de la intervención basada en el enfoque de atención primaria de salud para mejorar la capacidad de agencia de autocuidado, la adherencia al tratamiento, apoyo familiar, estrategias de afrontamiento ante el dolor crónico, y bienestar subjetivo en los pacientes con enfermedades crónicas no trasmisibles.

\section{Método}

Se trabajó con un diseño de tipo cuasiexperimental, ya que el enfoque que se empleó fue el de la psicoeducación. En este enfoque es común realizar comparativos de los programas de aprendizaje a toda la muestra sin emplear un grupo control (Alonso et al., 2016). De este modo se trabajó con el diseño pretest-postest, que evalúa distintas variables en una población con el fin de valorar cambios después de utilizar métodos que tengan el propósito de modificar variables subyacentes y manipuladas por el investigador (Little et al., 2019). El objetivo fue valorar el efecto de las variables de estudio antes y después de la aplicación del programa, para luego hacer su respectiva comparación estadística (Hernández et al., 2010).

\section{Participantes}

El tipo de muestreo fue intencional, debido a que se seleccionaron a las personas que tienen posibilidad de formar parte de la muestra debido al acceso que se tiene de los pacientes de acuerdo con los criterios de inclusión que implicaron: ser pacientes mayores de edad que estuvieran recibiendo tratamiento por alguna enfermedad crónica 
no trasmisible y que desearon participar voluntariamente en el programa. Cabe mencionar que las personas analfabetas fueron auxiliadas en el llenado de las cédulas e instrumentos correspondientes, por lo que la escolaridad no fungió como criterio de exclusión en ningún caso.

Se trabajó con pacientes con enfermedades crónicas no trasmisibles como la obesidad, hipertensión, diabetes, cáncer de mama y factores de riesgo como las adicciones y el sobrepeso que reciben tratamiento en alguno de los hospitales del Instituto de Salud del Estado de México (ISEM) de la ciudad de Toluca, estado de México. La muestra estuvo conformada por 188 personas, 53 hombres y 135 mujeres. La edad de los participantes osciló entre los 20 y 64 ańos de edad, con una media de 34.68 años y una desviación estándar de 8.00. Tienen las siguientes actividades laborales: 105 personas fueron amas de casa, 17 obreros, 16 empleados, 11 albañiles, 9 técnicos, 6 servidores públicos, 5 estudiantes, 5 comerciantes, 5 desempleados, 4 docentes, 3 choferes y 2 secretarias. En cuanto al nivel educativo, el $79 \%$ contaba con educación básica, el 16\% con nivel medio superior y el 5\% con licenciatura.

\section{Medición}

Se utilizó la escala para la valoración de la capacidad de agencia de Autocuidado (Manrique et al., 2009) validada para muestras mexicanas (Araujo et al., 2013), cuyo objetivo fue evaluar la capacidad de agencia de autocuidado en las categorías de interacción social, consumo suficiente de alimentos, bienestar personal, promoción del funcionamiento y desarrollo personal y actividad y reposo. Esta escala tiene un total de 24 ítems con un formato de respuesta de 5 alternativas tipo Likert, donde el 1 (totalmente en desacuerdo) significa el valor más bajo de capacidad de agencia de autocuidado y el 5 (totalmente de acuerdo) el más alto. Cada individuo puede obtener un puntaje que va de 24 a 120 puntos. La validez se obtuvo a través de un análisis factorial con rotación varimax y extracción de componentes principales e indica una estructura comprendida por 9 factores: Adquisición de técnicas de autocuidado, Prevención de riesgos que pongan en peligro la vida, Función de los grupos sociales, Equilibrio de la dieta, Búsqueda de ayuda, 
operacionalización de las actividades para el autocuidado, mejoramiento autocuidado, equilibrio entre actividad física y descanso y comunicación social. que explican $65.87 \%$ de la varianza total. La confiabilidad del instrumento presenta un alfa de Cronbach de .74 .

Asimismo, se hizo uso de una Escala de Adherencia al tratamiento (Salinas, et al., 2014) diseñada en México. Su objetivo es determinar la Adherencia al tratamiento en pacientes con enfermedades crónicas. La escala consiste en 24 reactivos con 3 alternativas de respuesta (siempre, algunas veces y nunca). La validez se obtuvo a través del análisis de varianza con rotación varimax y extracción de componentes principales con Kaiser, arrojó 7 dimensiones que explican el 58.76\% del total de la varianza: Apoyo del personal y familia, Confianza en personal salud, Percepción de dificultades, Percepción de vulnerabilidad, Cumplimiento de la dieta, Opinión externa en tratamiento y Autoaceptación de competencia del tratamiento. La confiabilidad se analizó con la prueba alfa de Cronbach, obteniéndose un índice de .72.

El apoyo familiar fue medido a través de la escala de apoyo social percibido de la familia (Domínguez et al., 2010), que cuenta con una varianza total explicada para la escala de apoyo social percibido de la familia (PSS-Fa) de $48.5 \%$ y un alfa de Cronbach de .87 al ejecutar la prueba que busca evaluar el apoyo social vinculado a las relaciones familiares; Presenta 16 ítems con un formato de 3 respuestas: Sí, No y No sé, otorgando la opción: Sí y 0 a las restantes opciones: No y No sé. Cabe mencionar que esta escala es parte de un instrumento denominado "Escala de apoyo social percibido por la familia y amigos", y se utilizó solamente el factor familia para fines de esta investigación.

También se utilizó el Cuestionario de estrategias de afrontamiento ante el dolor crónico (Soriano \& Monsalve, 2002), una escala para medir las estrategias de afrontamiento ante el dolor crónico con 5 opciones de respuesta: Totalmente en desacuerdo $=0$, Más en desacuerdo que de acuerdo $=1$, Ni de acuerdo ni en desacuerdo $=2$, Más de acuerdo que en desacuerdo $=3$ y Totalmente de acuerdo $=4$. Se adaptó el cuestionario para población mexicana con 23 ítems que explicaron el 64\% de la varianza total y un alfa de Cronbach de .83 mediante las técnicas de 
evaluación por jueces, entrevistas cognitivas, análisis factorial exploratorio y confirmatorio con una muestra diferente de la que fue estudiada en esta investigación. Se conservaron todos los factores originales con: 4 reactivos para creencias, 3 para autoafirmación, 3 de autocontrol mental, 3 de búsqueda de información, 3 de distracción y 4 de catarsis.

Asimismo, se utilizó la Escala de bienestar subjetivo (González, 2013) que mide el bienestar subjetivo en personas entre 15 y 45 años, basados en indicadores subjetivos y objetivos. La prueba consta de 29 ítems con 4 opciones de respuestas: totalmente de acuerdo, de acuerdo, en desacuerdo y totalmente en desacuerdo. Los factores de la escala son: crecimiento personal (6 ítems), relaciones positivas con otros (5 ítems), proyecto de vida (4 ítems), autoaceptación ( 4 ítems), planes a futuro (4 ítems), rechazo personal (3 ítems) y control personal (3 ítems). Se obtuvieron valores alfa por escala van de .74 a .83 y Se explica el $60.51 \%$ de la varianza total.

\section{Procedimiento}

El enfoque de Atención Primaria de Salud contempla a la salud como un derecho humano, más allá de lo político y social y pone énfasis en las actividades para conseguir mejoras en cualquier ámbito que contemple la salud humana y que sean equitativas para la toda la población (Starfield \& Aspachs, 2011). Con base en este enfoque se desarrolló un programa de educación en salud.

En este trabajo se desarrolla un programa de educación en salud basado en el enfoque de Atención Primaria de Salud para atender a las necesidades de los pacientes con las ECNT que más preocupan al sistema de salud actual como son la diabetes mellitus tipo 2, obesidad, hipertensión arterial sistémica y EPOC con una duración de 14 semanas.

Tomando en cuenta las variables estudiadas por diversos autores que han dedicado tiempo al estudio de las ECNT, se hizo una revisión cuidadosa para seleccionar los temas a desarrollar en el programa. Se dedican algunas páginas a la descripción de este análisis y posteriormente se presentan las cartas descriptivas que contienen la información 
de los objetivos de cada una de las sesiones, el tiempo de cada actividad, actividades a desarrollar, contenidos didácticos, material de apoyo y evaluación al final de cada sesión.

Para determinar los temas a incluirse en el programa, se llevó a cabo un análisis de los factores de instrumentos usados para medir las variables de Autocuidado, Adherencia al tratamiento, Apoyo social percibido por la familia y amigos, afrontamiento al dolor crónico y bienestar subjetivo como se muestra a continuación:

1. La Escala para la valoración de la capacidad de agencia de Autocuidado (Manrique et al., 2009) está conformada por los 9 factores que se enlistan a continuación:

1.1. Adquisición de técnicas de autocuidado

1.2. Prevención de peligros contra la vida, funcionamiento y bienestar humanos

1.3. Prevención del funcionamiento y desarrollo humano entre grupos sociales

1.4. Equilibrio en el mantenimiento de una ingesta suficiente de comida

1.5. Búsqueda de ayuda para la conservación de técnicas de autocuidado

1.6. Operacionalización de técnicas de autocuidado

1.7. Mejoramiento de las técnicas de autocuidado

1.8. Equilibrio entre actividad y descanso

1.9. Equilibrio entre soledad y comunicación social

2. La escala de adherencia al tratamiento (Salinas et al., 2014) tiene los siguientes 7 factores:

2.1. Apoyo del personal de salud y familiares

2.2. Relación de confianza con el personal de salud

2.3. Percepción de dificultades para llevar a cabo el tratamiento

2.4. Percepción de vulnerabilidad y beneficios del tratamiento

2.5. Cumplimiento de dieta e indicaciones médicas

2.6. Opinión de otros para el seguimiento del tratamiento indicado

2.7. Autoaceptación de la competencia para llevar el tratamiento 
3. La escala de apoyo social percibido de la familia y amigos (Domínguez et al., 2010) tiene solamente los dos siguientes factores:

3.1. Apoyo social percibido de la familia

3.2. Apoyo social percibido de los amigos

4. El cuestionario de afrontamiento al dolor crónico (Soriano \& Monsalve, 2002) está conformado por 6 factores, que son:

4.1. Creencias

4.2. Catarsis

4.3. Distracción

4.4. Autoafirmación

4.5. Autocontrol mental

4.6. Búsqueda de información del padecimiento

5. El cuestionario de Bienestar Subjetivo (González, 2013) consta de 6 factores:

5.1. Control personal

5.2. Rechazo personal

5.3. Planes a futuro

5.4. Autoaceptación

5.5. Propósito de vida

5.6. Crecimiento personal

A partir de las dimensiones de cada instrumento, se buscaron los temas a desarrollar que abarcaran cada una de estas y se elaboraron las cartas descriptivas para 14 sesiones de 3 horas cada una, que comprendió el programa de educación en salud, contemplando dos sesiones para las dos mediciones necesarias. Posteriormente se les dio un nombre sin tecnicismos y con un lenguaje apropiado para cualquier tipo de población. Los temas fueron los siguientes: 1.-Medición pretest, 2.-La participación social en el mi entorno, 3.-Conservo mi salud a través del autocuidado, 4.-¿Dónde busco información de mi padecimiento?, 5.-Cuido mi salud a través del plato del buen comer y la jarra del buen beber, 6.-El ejercicio y descanso para estar saludable, 7.-Estrategias para cumplir con mi tratamiento, 8.-Taller para 
la elaboración de autobiografía y plan de vida, 9.-Ejercicio vivencial de autoestima, 10.-Técnicas de control mental, 11.-Mi árbol genealógico, 12.-Estrategias para enfrentar mis malestares, 13.-Dinámica para observar mi desarrollo personal y 14.-Medición postest. El documento fue sometido a un análisis por parte de jueces expertos en salud pública y pedagogía quienes hicieron las observaciones correspondientes antes de la aplicación del programa. Se realizó una invitación en las instituciones de salud pública, sitios concurridos, oficinas gubernamentales y escuelas para que participaran en los talleres de educación en salud, acordando las fechas y horarios previamente con los responsables de cada sección municipal.

Se trabajó con un total de 10 grupos experimentales de 25 personas cada uno en el que se comprometieron al 100\% de las asistencias en el trascurso de las 16 semanas en las que se desarrolló la investigación. Se realizaron listas de asistencia y a pesar de que en un principio se contó con 300 personas para participar en la investigación, finalmente se contemplaron a 250 personas (divididas en los 10 grupos antes mencionados) para fines estadísticos. El resto de los participantes se vio beneficiado de los talleres que se llevaron a cabo y realizaron el postest pero por cuestiones de exlusión como fueron inasistencias, retardos o que no pudieron llenar adecuadamente los instrumentos a pesar de que se les auxilió para completarlos.

Se convocó a los pacientes de distintas instituciones de salud que residían en diversos municipios que pertenecen al Valle de Toluca. En cada municipio se prepararon los espacios necesarios como las aulas con el mobiliario preciso y se adquirieron los equipos y herramientas para realizar el programa de atención primaria de salud. En todos los casos se llevó a cabo la logística, que consistió en la reproducción del material para los pacientes. Se adaptaron los espacios para colocar el proyector, bocinas y computadora para la presentación de diapositivas.

Todo comenzó puntualmente y la primera semana en cada sitio, se llevó a cabo la aplicación del pretest. Al finalizar todas las sesiones, se citó a los pacientes para recoger su constancia de participación y ahí se aplicó el postest 4 meses después del pretest. Finalmente se compararon 
ambos resultados para medir los cambios ocurridos en cada una de las variables. La eficiencia del programa se evaluó después de comparar los resultados del pretest contra los del postest.

Todos los participantes tuvieron información suficiente acerca de la naturaleza de la investigación, existiendo para ello una plática informativa previa a las mediciones y aplicación del programa, además de proporcionarles por escrito una carta de consentimiento informado en la que se detallan los objetivos, riesgos, complicaciones y temas que se abordaron durante el desarrollo de los talleres.

De la misma manera, se les entregó el aviso de la confidencialidad de sus datos personales y una carta de consentimiento informado, conforme a los principios éticos que marca la Declaración de Helsinki (Asociación Médica Mundial, 2013), en donde se estipula que toda la información otorgada desde el inicio de la intervención será tratada de manera privada y únicamente para fines investigativos. La participación de todas las personas que decidieron formar parte de la muestra fue de forma voluntaria y no se trabajó con pacientes menores de edad. Durante el desarrollo de los talleres se procuró un ambiente inclusivo, de responsabilidad en el cuidado de su salud y el respeto al valor fundamental de la dignidad y de la vida de todos los participantes.

\section{Análisis de datos}

Para el análisis estadístico se usó la prueba estadística de la t de Student para muestras relacionadas, prueba estadística en la que se exige dependencia entre las variables, que, en un primer período, las observaciones servirán de control para conocer los cambios que se susciten después de aplicar una variable experimental (González \& Pérez, 2012).

\section{Resultados}

Como se puede observar en la tabla 1, existe un puntaje mayor de las medias en la segunda medición con respecto a la primera, excepto en las variables de percepción de dificultades, percepción de vulnerabilidad 
y rechazo personal que son factores negativos, en donde el primer puntaje es el mayor. Y los valores de t y $p$ en cada una de las variables indican que estas diferencias son estadísticamente significativas. Existe una mejora en las mediciones de todos los instrumentos: autocuidado, adherencia terapéutica, creencias, catarsis, distracción, buisqueda de información, autoafirmación, autocontrol, apoyo familiar y bienestar.

En lo que concierne a las medias de los factores del instrumento de Autocuidado, el mejoramiento de autocuidado y equilibrio entre actividad física y descanso tienen más de 1.00 de diferencia. En los factores de Adherencia al tratamiento, la percepción de dificultades es la que muestra una diferencia significativa con 2.00. En las estrategias de afrontamiento, los factores creencias, catarsis, distracción y autocontrol son los que presentaron más cambios. Por otro lado, el factor de Autoaceptación del tratamiento y el apoyo familiar de los instrumentos de Adherencia terapéutica y Apoyo social percibido de la familia, respectivamente, son los que sufrieron menos cambios.

Con respecto al tamaño del efecto, las variables de autocuidado, estrategias de afrontamiento ante el dolor crónico y bienestar subjetivo tienen factores que se encuentran por encima de dos desviaciones estándar en el postest, en comparación con el pretest. En lo referente al autocuidado, los puntajes de los factores de mejoramiento de autocuidado y equilibrio entre actividad física y descanso tuvieron mayor efecto. Lo mismo ocurre con todas las estrategias de afrontamiento ante el dolor crónico, excepto buisqueda de información. En relación con el bienestar subjetivo el mayor efecto lo tuvieron los factores de autoaceptación, planes a futuro y control personal. 
Efecto de un programa de atención primaria de salud en pacientes mexicanos / Domínguez et al.

\section{Tabla 1}

Medias y desviaciones estándar de los instrumentos aplicados

\begin{tabular}{|c|c|c|c|c|c|c|c|}
\hline Factores & $\begin{array}{l}\text { Media } \\
\text { (antes) }\end{array}$ & $\begin{array}{c}\text { D.E. } \\
\text { (antes) }\end{array}$ & $\begin{array}{c}\text { Media } \\
\text { (después) }\end{array}$ & $\begin{array}{c}\text { D.E. } \\
\text { (después) }\end{array}$ & $\mathrm{t}$ & $p$ & d \\
\hline \multicolumn{8}{|c|}{ Capacidad de agencia de autocuidado } \\
\hline Adquisición de técnicas & 3.701 & .901 & 4.557 & 0.751 & 16.971 & 0.00 & 1.750 \\
\hline Prevención peligro vida & 3.729 & .933 & 4.593 & 0.235 & 19.175 & 0.00 & 1.978 \\
\hline Función grupos sociales & 3.919 & .912 & 4.638 & 0.687 & 13.817 & 0.00 & 1.425 \\
\hline Equilibrio dieta & 3.903 & .983 & 4.582 & 0.762 & 14.145 & 0.00 & 1.459 \\
\hline Búsqueda de ayuda & 3.706 & 1.031 & 4.539 & 0.829 & 15.623 & 0.00 & 1.611 \\
\hline Operacionalización & 3.884 & .982 & 4.622 & 0.732 & 16.602 & 0.00 & 1.712 \\
\hline Mejoramiento autocuidado & 2.947 & .623 & 4.143 & 0.644 & 24.431 & 0.00 & 2.520 \\
\hline Eq. Actividad física-descanso & 2.611 & .932 & 3.904 & 0.781 & 22.972 & 0.00 & 2.369 \\
\hline Comunicación social & 3.383 & 1.041 & 4.371 & 0.772 & 16.942 & 0.00 & 1.747 \\
\hline \multicolumn{8}{|c|}{ Adherencia al tratamiento } \\
\hline Apoyo del personal y familia & 2.672 & .161 & 2.838 & 0.228 & 6.744 & 0.00 & 0.862 \\
\hline Confianza en personal salud & 2.877 & .193 & 2.983 & 0.221 & 5.002 & 0.00 & 0.516 \\
\hline Percepción dificultades & 3.257 & 3.866 & 1.134 & 0.8274 & 6.744 & 0.00 & 0.696 \\
\hline Percepción vulnerabilidad & 2.760 & .906 & 2.345 & 0.661 & 5.783 & 0.00 & 0.596 \\
\hline Cumplimiento de la dieta & 2.681 & .343 & 2.956 & 0.275 & 8.441 & 0.00 & 0.870 \\
\hline $\begin{array}{l}\text { Opinión externa en } \\
\text { tratamiento }\end{array}$ & 1.733 & .242 & 1.962 & 0.177 & 10.732 & 0.00 & 1.107 \\
\hline $\begin{array}{l}\text { Autoaceptación del } \\
\text { tratamiento }\end{array}$ & 2.894 & .312 & 2.964 & 0.245 & 2.837 & 0.01 & 0.293 \\
\hline
\end{tabular}

Estrategias de afrontamiento ante el dolor crónico

\begin{tabular}{lccccccc} 
Creencias & 2.943 & 1.078 & 4.101 & 0.839 & 22.441 & 0.00 & 2.315 \\
Catarsis & 3.196 & 1.063 & 4.217 & 0.826 & 23.903 & 0.00 & 2.465 \\
Distracción & 3.228 & 1.065 & 4.232 & 0.842 & 20.461 & 0.00 & 2.110 \\
Búsqueda de Información & 3.782 & .936 & 4.504 & 0.754 & 17.145 & 0.00 & 1.768 \\
Autoafirmación & 3.525 & .932 & 4.382 & 0.693 & 23.016 & 0.00 & 1.768 \\
Autocontrol & 2.934 & .905 & 4.125 & 0.725 & 25.792 & 0.00 & 2.660 \\
\hline
\end{tabular}




\begin{tabular}{|c|c|c|c|c|c|c|c|}
\hline Factores & $\begin{array}{l}\text { Media } \\
\text { (antes) }\end{array}$ & $\begin{array}{c}\text { D.E. } \\
\text { (antes) }\end{array}$ & $\begin{array}{c}\text { Media } \\
\text { (después) }\end{array}$ & $\begin{array}{l}\text { D.E. } \\
\text { (después) }\end{array}$ & $\mathrm{t}$ & $p$ & $\mathrm{~d}$ \\
\hline \multicolumn{8}{|c|}{ Apoyo social percibido de la Familia } \\
\hline Apoyo Familiar & 2.772 & .141 & 2.867 & 0.058 & 9.303 & 0.00 & 0.960 \\
\hline \multicolumn{8}{|c|}{ Bienestar subjetivo } \\
\hline Crecimiento personal & 3.818 & .308 & 3.975 & 0.112 & 8.08 & 0.00 & 0.833 \\
\hline Relaciones con otros & 3.375 & .443 & 3.847 & 0.246 & 18.50 & 0.00 & 1.908 \\
\hline Proyecto de vida & 3.776 & .364 & 3.964 & 0.154 & 7.52 & 0.00 & 0.776 \\
\hline Autoaceptación & 3.202 & .481 & 3.832 & 0.282 & 21.83 & 0.00 & 2.252 \\
\hline Planes a futuro & 3.393 & .287 & 3.897 & 0.218 & 25.00 & 0.00 & 2.579 \\
\hline
\end{tabular}

\section{Discusión}

Respecto al autocuidado, el desarrollo del programa hizo énfasis en la prevención de peligros contra la vida, mejorar la relación con los demás, el cuidado de la dieta, el ejercicio y el descanso con actividades que implicaron hablar de la opinión que tuvieron los pacientes de sí mismos, identificaron los beneficios y desventajas de su estilo que vida actual y la obtuvieron información acerca de la búsqueda de apoyo social. Los resultados muestran que hubo diferencias estadísticamente significativas en los datos obtenidos antes y después de la intervención y se contemplaron todos los factores del instrumento haciendo énfasis en: la adquisición, búsqueda, operacionalización y mejoramiento de técnicas de autocuidado. "El autocuidado propicia un cambio de hábitos en cuanto a la alimentación, actividad física y descanso en los pacientes con enfermedades crónicas" (Gallardo \& Monroy, 2016, p. 14), y además promueve la toma de responsabilidad sobre los padecimientos con los que tendrá que vivir una persona el resto de su vida. Junto con las estrategias de afrontamiento, la variable de autocuidado es la que muestra mayores modificaciones puesto que en todas las sesiones se hizo hincapié en la importancia de llevar a cabo acciones para el cuidado personal. 
En cuanto a la adherencia al tratamiento, se habló de la importancia de las terapias farmacológicas y no farmacológicas, la asistencia a consultas, el respeto de los tiempos y horarios de las citas, la necesidad de confiar en el personal de salud y llevar una dieta conforme a las prescripciones nutricionales aunque eso implicara buscar la ayuda de sus familiares. Conforme a los resultados, se observan diferencias significativas estadísticamente en los datos obtenidos antes y después de la intervención. Los factores que se abordaron fueron: la importancia de relación de confianza con el personal de salud, cumplimiento de dieta $e$ ingesta suficientes, beneficios de llevar a cabo el tratamiento y equilibrio entre actividad fisica y descanso. Es posible comprobar la relación entre la adherencia terapéutica en pacientes con enfermedades crónicas y la mejora en los indicadores del estado de salud del individuo y advierten de la importancia de que el paciente se adhiera a las prescripciones del equipo de salud para recuperar el equilibrio del organismo (García \& Lucas, 2013). El factor que más modificaciones sufrió fue la importancia de la opinión externa en el tratamiento, es decir, que posterior a los talleres, los pacientes escucharon más a sus familiares en el sentido de recordatorios de hábitos saludables, horarios de tomas de medicamentos, entre otras opiniones respecto a las prescripciones médicas del paciente.

En lo que respecta al apoyo familiar, el programa se enfocó a la solicitud de ayuda a los familiares aun cuando hubiera vergüenza o miedo al rechazo. También se encontraron diferencias estadísticamente significativas en los datos obtenidos antes y después de la intervención que abordó el único factor del instrumento: apoyo social percibido por la familia y comunicación social. Se ha evaluado el bienestar en personas con enfermedades crónicas no trasmisibles en Perú y se afirma que está íntimamente ligado a la calidad de vida de los pacientes y a la recuperación de sus funcionalidades (Alpi et al., 2016). Los autores mencionan que "un paciente con suficiente apoyo familiar puede tener mayor bienestar en el transcurso de su vida, pues mediante el cuidado y afecto de los seres queridos mejoran los estados de ánimo y psicológico de los enfermos" (Alpi et al., 2016, p. 176). También se ha señalado que 
la falta de apoyo familiar para los pacientes con enfermedades crónicas da como resultado el estrés para el paciente y sus cuidadores además de que la institucionalización es más frecuente cuando se carece de la colaboración de la familia para proporcionar los cuidados más esenciales (Jiménez et al., 2017). Cabe mencionar que esta variable fue la que menos modificaciones tuvo dentro del programa, posiblemente porque las familias de los pacientes no estuvieron implicadas en el trascurso de las sesiones.

Con relación al afrontamiento ante el dolor crónico, se encontraron diferencias significativas estadísticamente en los datos obtenidos antes y después de la intervención en la que se tomaron en cuenta cada uno de los factores del instrumento y se midió cada estrategia: creencias, catarsis, distracción, búsqueda de información, autoafirmación y autocontrol mental. La adquisición de herramientas que fortalezcan la capacidad de afrontamiento en las personas que transcurren por una enfermedad crónica, contribuye a la vida digna y al bienestar de dichos pacientes (Jiménez et al., 2017). La estrategia que mayor incremento tuvo fue autocontrol, seguida de catarsis, autoafirmación, creencias, distracción y búsqueda de información. Este último factor fue el que menos diferencias mostró en parte porque no existió una actividad extra-clase en la que los pacientes buscaran información de sus padecimientos.

En lo que concierne al programa respecto al bienestar subjetivo, el participante realizó su autobiografía y mediante unas preguntas estructuró su plan de vida hasta obtenerlo como una redacción que leyó frente a sus compañeros. Al final de todos los talleres cada asistente que concluyó el programa recibió un reconocimiento y se realizó una convivencia en donde pudieron exponer los avances que subjetivamente apreciaban. En esta variable se encontraron diferencias estadísticamente significativas, mediante la prueba t de Student, en los datos obtenidos antes y después de la intervención que manejó factores como: Desarrollo y bienestar humano, crecimiento personal, planes a futuro y propósito de vida. La percepción negativa del bienestar en las personas con padecimientos crónicos es predictora de complicaciones a largo plazo pudiendo incluso generar estilos de vida poco saludables 
que lleven a los adultos enfermos a presentar más de una enfermedad crónica o pluripatología (Jiménez, 2012). De ahí la importancia en el abordaje de estos temas en programas de salud enfocados a personas con ECNT. Los factores en donde hubo mayor diferencia fueron los planes a futuro y la autoaceptación que concuerdan más directamente con las dinámicas que se llevaron a cabo. Sin embargo, es recomendable una revisión en las actividades de los temas de proyecto de vida y crecimiento personal, debido a que fueron los factores que donde menor incremento hubo.

Las limitantes para este estudio se presentaron desde la recolección de la muestra que se determinó de manera intencional, pues acudieron al llamado todas aquellas personas interesadas en la mejora de su salud. Asimismo, en todos los municipios hubo pacientes que no completaron el $100 \%$ de las sesiones, por lo que tuvieron que ser excluidos para el análisis de los resultados. De la misma forma ocurrió con quienes no cubrieron con los requisitos para formar parte de la muestra. Se preveía mayor número de participantes en todos los municipios e incluso llegar a algunas localidades donde fue imposible el acceso para los investigadores debido a obstáculos burocráticos.

A pesar de haber planeado de manera precisa las actividades del programa, hubo variables extrańas como no haber podido regresar a los lugares donde se aplicó el programa para dar un seguimiento más exhaustivo debido a la vigencia de los permisos, los recursos financieros y las características de la muestra.

Se sugiere tomar en cuenta las limitantes para el desarrollo de nuevas investigaciones acerca del tema y prever con suficiente antelación los permisos correspondientes con las autoridades políticas y sanitarias donde se pretenda llevar a cabo una investigación de esta índole.

Finalmente, se sugiere contemplar en estudios posteriores la intervención no solo con el paciente sino de ser posible con familiares que constituyen su red de apoyo más próxima. Seguramente se encontrarían datos de interés para la elaboración y desarrollo de programas de Atención Primaria de Salud. 


\section{Referencias}

Alonso, García-Sanz, León, García-Gordo, Gil y Ríos (2016). Métodos de investigación de enfoque experimental. International Journal of Morphology, International Journal of Morphology, 33(1), 382-387.

Alpi, S. V., Quiceno, J. M., y Munévar, F. R. (2016). Calidad de vida en adultos jóvenes colombianos según el sexo: Un estudio descriptivo comparativo. Salud y Sociedad, 6(2), 172-180. https:// doi.org/10.22199/s07187475.2015.0002.00005

Araujo, R., Acevedo-Treto, M., Román-Carrillo, M., Trejo-Ortiz, P., Mollinedo-Montaño, F. y Acuña, F. (2013). Capacidad de agencia de autocuidado como estilo de vida saludable en pacientes con hipertensión arterial sistémica. Memorias del $14^{\circ}$ Seminario de Investigación. Aguascalientes: Universidad Autónoma de Aguascalientes.

Asociación Médica Mundial (2013). Declaración de Helsinki de la Asociación Médica Mundial: Principios éticos para las investigaciones médicas en seres humanos. Brasilia: The World Medical Association.

Chauca, C., Sayda M., Yambay, C. y Verónica, I. (2018). Dimensiones de calidad de vida afectadas en los pacientes diabéticos. Distrito de salud Chambo-Riobamba, noviembre 2017-marzo 2018. 2018 (tesis de Licenciatura). Universidad Nacional de Chimborazo, Riobamba.

Domínguez, A., Salas, M., Contreras, C. y Procidano, F. (2010). Validez concurrente de la versión mexicana de las escalas de Apoyo Social Percibido de la Familia y los Amigos (PSS-Fa y PSS-Fr). Revista Latinoamericana de Psicología, 43(1), 125-137.

Etzwiler D. (2017) Don't ignore the patients. Diabetes Care, 24, 1840-1841. https://doi.org/10.2337/diacare.24.10.1840

Gallardo, L. y Monroy, G. (2016). El autocuidado y las apps, agentes de cambio en enfermedades como sobrepeso, obesidad y diabetes. Revista Digital Universitaria, 18(8), 1-13. 
García, P., y Lucas, V. (2013). Etapa de cambio y adherencia terapéutica en mujeres con enfermedades crónicas no transmisibles durante un programa de atención multidisciplinaria (tesis de doctorado inédita). Instituto Nacional de Salud Pública: México.

González, M. B. (2013). Prácticas parentales, bienestar psicológico y conductas de riesgo en adolescentes (tesis de doctorado inédita). Universidad Nacional Autónoma de México, México.

González ,M. y Pérez, A. (2012). Estadística aplicada: una visión instrumental. Madrid: Ediciones Díaz de Santos S.A.

Hernández, F., Fernández, C. y Baptista, L. (2010). Metodología de la investigación. México: McGraw-Hill.

Jiménez, E. S. (2012). Capacidad funcional y bienestar subjetivo de los pacientes geriátricos del municipio Cerro. 2012. Revista Cubana de Tecnología de la Salud, 2(2), 1-9.

Jiménez, E., Herrera, F., Sneider, A. y Arango, A. (2017). Afrontamiento de los pacientes con enfermedad crónica diabetes mellitus e Hta de la ESE Centro de Salud Ricaurte para el Año 2016 (tesis de doctorado inédita). Universidad de Cundinamarca, Fusagasugá, República de Colombia.

Jordan, E., Galea, G., Tukuitonga, C., Beaglehole, R. (2015). Preventing chronic diseases: taking stepwise action. Lancet, 366(1), 1667-1671. https://doi.org/10.1016/S0140-6736(05)67342-4

Lizaraso, C. y Ruiz, E. (2016). Hacia una nueva medicina preventiva. Horizonte Médico, 16(2), 4-5.

Little, T. D., Chang, R., Gorrall, B. K., Waggenspack, L., Fukuda, E., Allen, P. J., \& Noam, G. G. (2019). The retrospective pretest-posttest design redux: On its validity as an alternative to traditional pretest-posttest measurement. International Journal of Behavioral Development 23(1), 77-82.

Manrique, F., Fernández, A. y Velandia, A. (2009). Análisis factorial de la Escala para la valoración de la capacidad de agencia de Autocuidado (ASA). Aquichan, 9(3), 222-235.

Rivera, J., Barquera, S., Gonzalez-Cossio, T., Olaiz, G. y Sepulveda, J. (2014). Nutrition transition in Mexico and in other Latin 
American countries. Nutrition Reviews, 7(2), 149-157. https:// doi.org/10.1111/j.1753-4887.2004.tb00086.x

Rivera, T., Parra S. y Barriguete, J. (2015). Trastornos de la conducta alimentaria como factor de riesgo para la osteoporosis. Salud Pública de México, 47(4), 308-318. https://doi.org/10.1590/ S0036-36342005000400009

Romero, M., Shamah, T., Franco, A., Villalpando, S., Cuevas, L., Gutiérrez, J. P., Salinas, J., Espinoza V. y González H. (2014). Características psicométricas de una escala de adherencia al tratamiento de diabetes mellitus II e hipertensión en una muestra de pacientes. Revista Electrónica de Psicología Iztacala, 17(3), 905.

Mireles, S., Raygoza, N. y Jiménez, S. N. (2017). Comparación de la relación del estilo de vida con el sobrepeso y la obesidad en personas con y sin antecedentes heredofamiliares de diabetes tipo 2 . Jóvenes en la Ciencia, 3(2), 467-471.

Soriano, J. y Monsalve, V. (2002). CAD: Cuestionario de afrontamiento ante el dolor crónico (CAD). Revista de la Sociedad Española del Dolor, 9(1), 13-22.

Starfield, B. y Aspachs, J. P. (2011). Atención primaria: equilibrio entre necesidades de salud, servicios y tecnologia. Barcelona: Masson.

Strong, K., Mathers, C., Leeder, S. y Beaglehole, R. (2015). Preventing chronic diseases: how many lives can we save. Lancet, 366(9496), 1578-1582. https://doi.org/10.1016/S0140-6736(05)67341-2

Vignolo, J., Vacarezza, M., Alvarez, C. y Sosa, A. (2017). Niveles de atención, de prevención y atención primaria de la salud. Archivos de Medicina Interna, 34(1), 11-14.

Recibido: 22 de enero, 2019

Revisado: 6 de enero, 2020 Aceptado: 18 de enero, 2020 


\section{Gobierno del Estado de México}

Secretaría de Salud

Instituto de Salud del Estado de México

\section{Carta de Consentimiento Informado}

\begin{tabular}{|l|l|l|l|}
\hline UNIDAD MÉDICA & LUGAR & FECHA & HORA \\
\hline
\end{tabular}

\begin{tabular}{|c|c|c|c|}
\hline \multicolumn{3}{|c|}{$\begin{array}{l}\text { NOMBRE DEL PACIENTE (APELLIDO PATERNO, APELLIDO } \\
\text { MATERNO Y NOMBRE (S) }\end{array}$} & $\begin{array}{l}\text { NO. DE } \\
\text { EXPEDIENTE }\end{array}$ \\
\hline EDAD & $\begin{array}{l}\text { GÉNERO } \\
\text { \MASCULINO } \\
\text { 囚FEMENINO }\end{array}$ & OCUPACIÓN & ESTADO CIVIL \\
\hline \multicolumn{4}{|c|}{ DOMICILIO (CALLE, NÚMERO, COLONIA, LOCALIDAD, MUNICIPIO Y ESTADC } \\
\hline
\end{tabular}

Por medio de la presente acepto participar en el proyecto de investigación titulado: "EFECTO DE UN PROGRAMA DE ATENCIÓN PRIMARIA DE SALUD EN PACIENTES CON ENFERMEDADES CRÓNICAS NO TRASMISIBLES". En el hospital

del Instituto de salud del Estado de México.

El propósito de este estudio es medir el efecto de un programa de educación en salud basado en el enfoque de atención primaria de salud, destinado a la mejora su autocuidado, adherencia al tratamiento, apoyo familiar, afrontamiento al dolor crónico y bienestar subjetivo en pacientes del Instituto de salud del Estado de México (ISEM) con enfermedades crónicas no trasmisibles de la ciudad de Toluca, Estado de México.

Se me ha explicado que mi participación consistirá en: Responder los instrumentos de medición que me serán aplicados por una persona que se identifica como doctorante en Ciencias de la salud integrante del cuerpo académico de investigación "Socialización, juventud y estilos de crianza" de 
la Universidad Autónoma del Estado de México (UAEMéx), así como la participación en 12 talleres semanales con una duración de 3 hrs cada uno. El programa durará 4 meses en total.

Declaro que se me ha informado ampliamente sobre los posibles riesgos, inconvenientes, molestias y beneficios derivados de mi participación en el estudio, que son los siguientes:

Tengo como beneficio saber si este fenómeno está o pudiera estar afectando mi vida personal, familiar, laboral, entre otros, así como tener información actual y veraz respecto a los temas que tratará el programa.

$\mathrm{El}$ investigador principal me ha dado seguridades de que no se me identificará en las presentaciones o publicaciones que deriven de este estudio y de que los datos relacionados con mi privacidad serán manejados en forma confidencial, también se ha comprometido a proporcionarme la información actualizada que se obtenga durante el estudio, aunque esta pudiera hacerme cambiar de parecer respecto a mi permanencia en el mismo.

El investigador principal se ha comprometido a responder cualquier pregunta y aclarar cualquier duda que le plantee acerca de los procedimientos que se llevarán a cabo, los riesgos, beneficios o cualquier otro asunto relacionado con la investigación o con mi tratamiento (en caso que el proyecto modifique o interfiera con el tratamiento habitual del paciente el investigador se compromete a darme nformación oportuna sobre cualquier procedimiento alternativo adecuado que pudiera ser ventajoso para mi tratamiento).

Entiendo que conservo el derecho de retirarme del estudio en cualquier momento en que lo considere conveniente, sin que ello afecte la atención médica que recibo en el hospital mencionado anteriormente.

Mtro. Arturo Alejandro Domínguez López (Investigador principal) Cédula profesional: 09642836
NOMBRE Y FIRMA DEL PARTICIPANTE

Número telefónico al cual puede comunicarse en caso de emergencia, dudas o preguntas relacionadas con el estudio: (01722) 4870492 